Acta Cryst. (1995). D51, 819-823

\title{
Determination of Hemihedral Twinning and Initial Structural Analysis of Crystals of the Procarboxypeptidase A Ternary Complex
}

\author{
BY F. X. GoMIS-RÜTH* \\ Institut de Biologia Fonamental i Departament de Bioquímica, Universitat Autònoma de Barcelona-08193 \\ Bellaterra, Spain \\ I. FITA† \\ Department d'Enginyeria Química, ETS Enginyeria Industrial, Diagonal 647-08028 Barcelona, Spain
}

R. Kiefersauer AND R. Huber

Abteilung für Strukturforschung, Max-Planck-Institut für Biochemie, 82152 Martinsried, bei München, Germany

F. X. AVILÉS

Institut de Biologia Fonamental i Departament de Bioquímica, Universitat Autònoma de Barcelona-081983 Bellaterra, Spain

AND J. NAVAZA

UPR 180 CNRS Laboratoire de Physique, Centre Pharmaceutique, 92290 Chatenay Malabry, France

(Received 25 October 1994; accepted 13 January 1995)

\begin{abstract}
The initial structural analysis of the ternary complex of procarboxypeptidase A from hemihedrally twinned crystals diffracting up to $2.8 \AA$ is described. Detection of twinning by different techniques is presented, including biochemical and intensity statistics approaches. The structure was initially solved using Patterson-search techniques, and the three positioned search models were used to effectively deconvolute the twinned data.

\section{Introduction}

The ternary complex (TC) of the bovine digestive proenzyme procarboxypeptidase A (PCPA) with the serineproproteinases chymotrypsinogen $C$ (CTGC) and proprotease E (BPE) (Avilés, Vendrell, Guasch, Coll \& Huber, 1993) crystallizes in rhombohedral crystals of space group $R 3$ with $a=b=188$, and $c=82 \AA$ (hexagonal setting). These crystals display twinning by hemihedry, being the crystal composed by two differently orientated twin domains (Koch, 1992; Redinbo \& Yeates, 1993). The relative fractions of both twin species are $\alpha$ and $(1-\alpha)$, respectively [ $\alpha$ is referred to as the fraction of the less abundant domain related to the entire

\footnotetext{
* Current address: Abteilung für Strukturforschung, Max-PlanckInstitut für Biochemie, 82152 Martinsried, Germany.

$\dagger$ Corresponding author.

crystal volume $(0 \leq \alpha \leq 0.5)$ ]. This intrinsic problem has been often observed and discussed in depth (Fisher \& Sweet, 1980; Rees, 1980; Redinbo \& Yeates, 1993).

In the present case, the twinning symmetry operator is a twofold axis along [110], the diagonal between $\mathbf{a}$ and $\mathbf{b}$, giving rise to a diffraction pattern that simulates $R 32$ symmetry. This means that the twin-related reflections are those of indices $(h k l)$ and $(k h-l)$, respectively, with associated intensities $I_{h k l}^{\text {obs-theo }}$ and $I_{k h-l}^{\text {obs-theo }}$. The resulting intensities $I_{h k l}^{\text {obs }}$ and $I_{k h-l}^{\text {obs }}$ in the diffraction pattern are therefore composed as described in (1) and (2).

$$
\begin{aligned}
& I_{h k l}^{\text {obs }}=\left[(1-\alpha) I_{h k l}^{\text {obs-theo }}\right]+\left(\alpha I_{k h-l}^{\text {obs-theo }}\right), \\
& I_{k h-l}^{\text {obs }}=\left(\alpha I_{h k l}^{\text {obs-theo }}\right)+\left[(1-\alpha) I_{k h-l}^{\text {obs-theo }}\right] .
\end{aligned}
$$

This linear system of equations can, in principle, be solved whenever $\alpha \neq 0.5$. However, some severe problems arise in practice. First, the system is very dependent on a good estimation of $\alpha$. Rees (1980) studied this problem extensively. According to this analysis, it is possible to estimate $\alpha$ using Wilson's diffraction intensity distributions (Wilson, 1949). A cumulative distribution function $N(Z, \alpha)$, where $Z$ represents the intensity relative to the mean intensity, gives the fraction of reflections having an intensity less than $Z$. This function, for non-centrosymmetric reflec- 
tions, is given by (3) and (4),

$$
\begin{aligned}
N(Z, \alpha)= & (\alpha[\exp (-Z / \alpha)-1]-(1-\alpha) \\
& \times\{\exp [-Z /(1-\alpha)]-1\}) /[(1-2 \alpha)],
\end{aligned}
$$

for $\alpha \neq 0.5$,

$$
N(Z, \alpha)=1-(1+2 Z) \exp (-2 Z), \text { for } \alpha=0.5 \text {. }
$$

The graphic representation $Z$ against $N(Z, \alpha)$ renders a curve with exponential shape for $\alpha=0.0[N(Z, 0)=$

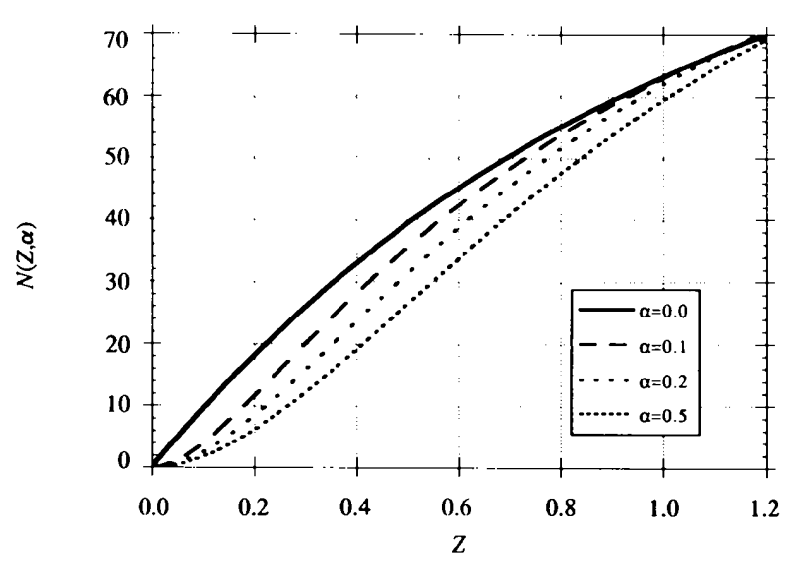

(a)

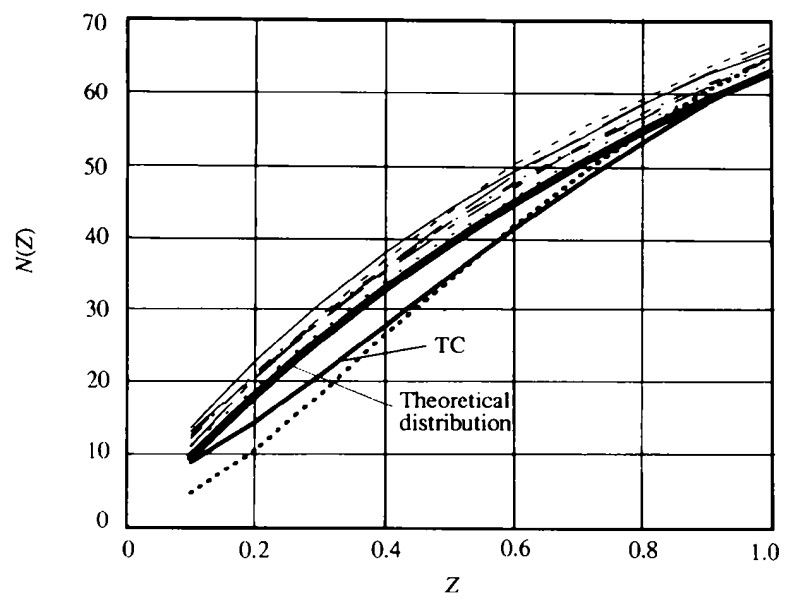

(b)

Fig. 1. (a) Graphical representation for different twinning fractions $\alpha$ of the theoretical cumulative distribution function $N(Z, \alpha)$, where $Z$ represents the intensity relative to the mean intensity, giving the fraction of reflections having an intensity less than $Z$. This function, for non-centrosymmetric reflections of crystals, is given by $(3)$ and (4) in the text. Note that $N(Z, \alpha)=N[Z,(1-\alpha)]$. (b) Experimental $N(Z)$ curves. A comparison with the theoretical distribution for $\alpha=0.0$ (thickest, solid line) as a reference is included. Six untwinned data sets arbitrarily chosen (R. Huber, unpublished results; thin lines) display an exponential habitus and are always located above the theoretical distribution for an untwinned crystal. TC data (thick, solid line), so as a further data set (R. Huber, unpublished results; thick dashed line) for which twinning was suspected, render a sigmoid habitus and lie below the theoretical values. $1-\exp (-Z)]$ and a progressively sigmoid one for increasing values of $\alpha$, as observed in Fig. 1(a). However, it may be difficult to obtain a quantitative estimation of the twinning degree in experimental data just by comparing the experimental $N(Z)$ curves with the theoretical ones, as discussed later (Fig. 1b). In any case, the clearly different habitus of the curve obtained from untwinned data may allow at least to confirm or discard twinning.

Even when an accurate value of $\alpha$ is available, the solution of the linear system of (1) and (2) amplifies errors in intensity determinations by a factor of approximately $1 /(1-2 \cdot \alpha)$ (Redinbo \& Yeates, 1993). Thus, the error grows as $\alpha$ increases and becomes infinity for perfect twinning $(\alpha=0.5$ ), when the system given by (1) and (2) is singular.

Another problem when trying to solve (1) and (2) arises from the fact that only data $I_{h k l}^{\text {obs-theo }}$, for which the corresponding twin partners $I_{k h-l}^{\mathrm{obs}}$ and $I_{h k l}^{\mathrm{obs}}$ are present, may be determined. Thus, the amount of missing data in the deconvoluted spectrum will be about twice the missing experimental data.

In this work, we report the initial resolution of the crystal structure of the ternary complex (TC) of procarboxypeptidase A using twinned crystals and Patterson-search techniques. A method to accurately determine the twin fraction $\alpha$ when a partial solution is available is also discussed.

\section{Detection of hemihedral twinning}

Crystal diffraction data up to $2.8 \AA$ resolution for TC were collected from a single crystal on an MAR Research image-plate area detector attached to a Rigaku-Denki generator producing $\mathrm{Cu} K \alpha$ radiation operated at $5.4 \mathrm{~kW}$. The data were processed with MOSFLM (Leslie, 1991) and gave an internal agreement factor $R_{\text {symm }}$ of $7.6 \%$ after evaluation with PROTEIN (Steigemann, 1991), when using the space group $R 3$, and of $14 \%$ with $R 32$. Thus, both possibilities were considered at this point, real space group symmetry $R 32$ or $R 3$ with strong hemihedral twinning.

With one TC oligomer per asymmetric unit, we calculate $V_{m}$ of $2.7 \AA^{3} \mathrm{Da}^{-1}$ for $R 3$ or $1.3 \AA^{3} \mathrm{Da}^{-1}$ for $R 32$ (Matthews, 1968). Of these, only the value for $R 3$ seems acceptable. The crystallization conditions could have provoked the (at least partial) dissociation of the complex, giving in fact crystals of a binary complex (PCPA + BPE or PCPA + CTGC) or even, though unlikely, a mixture of both binary complex types, as the sequences of the two proteases BPE and CTGC are closely related (Kerfelec, Cambilleau, Puigserver \& Chapus, 1986; Pascual et al., 1990). For the binary complexes, the $V_{m}$ value would be $2.0 \AA^{3} \mathrm{Da}^{-1}$ for $R 32$, clearly within the acceptable range (Matthews, 1968; Bode \& Schirmer, 1985). Therefore, the presence of the 
Table 1. Analysis of the crystal volume $\left(V_{K R}\right)$ determination as applied according to the method developed by Kifersauer and coworkers (1995) to five crystals of the ternary complex of procarboxypeptidase $A(a)$ to $(e)$

\begin{tabular}{|c|c|c|c|c|c|c|}
\hline & (a) & (b) & $\begin{array}{l}\text { Crystal } \\
(c)\end{array}$ & (d) & (e) & \\
\hline Estimated volume (nl) & 3.10 & 4.28 & 8.11 & 3.60 & 4.18 & \\
\hline $\begin{array}{l}\text { Protein contents (PQ) (pmol) } \\
\text { per crystal considering different } \\
\text { types of complexes }\end{array}$ & & & & & & \\
\hline PCPA + CTGC + BPE & 20 & 28 & 47 & 21 & 22 & \\
\hline $\begin{array}{c}(\mathrm{PCPA}+\mathrm{CTGC})+(\mathrm{PCPA}+\mathrm{BPE}) \\
\text { (combination of binary complexes) }\end{array}$ & 13 & 19 & 32 & 14 & 15 & \\
\hline $\mathrm{PCPA}+\mathrm{BPE}$ & 28 & 40 & 65 & 29 & 30 & \\
\hline $\mathrm{PCPA}+\mathrm{CTGC}$ & 28 & 40) & 65 & 29 & 30) & \\
\hline $\begin{array}{l}\text { Number of protein complexes }(n) \text { within } \\
\text { the asymmetric unit considering different } \\
\text { complexes }\end{array}$ & & & & & & $\begin{array}{l}\text { Average } \\
(a)-(e)\end{array}$ \\
\hline $\mathrm{PCPA}+\mathrm{CTGC}+\mathrm{BPE}$ & 1.08 & 1.10 & 0.97 & 0.98 & 0.88 & $1.00 \pm 0.12$ \\
\hline $\begin{array}{r}(\mathrm{PCPA}+\mathrm{CTGC})+(\mathrm{PCPA}+\mathrm{BPE}) \\
\text { (combination of binary complexes) }\end{array}$ & 0.75 & 0.75 & 0.66 & 0.65 & 0.60 & $0.67 \pm 0.08$ \\
\hline $\mathrm{PCPA}+\mathrm{BPE}$ & 1.52 & 1.57 & 1.35 & 1.35 & 1.21 & $1.40 \pm 0.19$ \\
\hline PCPA + CTGC & 1.52 & 1.57 & 1.35 & 1.35 & 1.21 & $1.40 \pm 0.19$ \\
\hline
\end{tabular}

TC in the crystals was experimentally verified: (i) crystals were carefully washed and dissolved in $2 M$ urea. $\mathrm{N}$-terminal chemical sequencing of this protein solution showed, unambiguously, the presence of the three molecules in approximately equimolar ratios. (ii) The procedure developed by Kiefersauer and coworkers (Kiefersauer, Stetefeld, Lottspeich \& Huber, 1995) in order to determine the volume of single crystals was applied in combination with a quantitative amino-acid composition determination. Five crystals were analyzed in this way, testing different hypothetical complexes in the asymmetric unit. (5), transformed according to our case, was applied,

$$
\begin{aligned}
n & =\left(N_{\mathrm{PR}}\right) /\left[z\left(V_{\mathrm{KR}}\right) /\left(V_{\mathrm{UC}}\right)\right] \\
& =\left(P Q \times 6.023 \times 10^{11}\right) /\left[9\left(V_{\mathrm{KR}}\right) /\left(V_{\mathrm{UC}}\right)\right],
\end{aligned}
$$

where $n$ is the number of (binary or ternary) complex units present in the asymmetric unit, $N_{\mathrm{PR}}$ is the number of protein oligomers in the crystal, $z$ is the number of asymmetric units per cell (nine was taken; the possibility of a hypothetical $R 32$ space group was analyzed assuming two binary complexes in the asymmetric unit), $V_{\mathrm{KR}}$ (in nanolitres) is the measured crystal volume, $V_{\mathrm{UC}}$ is the volume of the unit cell $\left(2.51 \times 10^{-12} \mathrm{nl}\right)$, and $\mathrm{PQ}$, the protein quantity, is given in pmol. The results are presented in Table 1 , showing that only one ternary complex per asymmetric unit $(n=1)$ is compatible with the measured data. The pseudo-twofold symmetry is therefore due to hemihedral twinning.

A further evidence for the presence of twinning in the TC crystals was obtained by analyzing the experimental cumulative distribution function $N(Z)$, as indicated before (Fig. 1b). In this analysis, the curve obtained from the experimental diffraction data of a TC crystal shows clear sigmoidal shape when compared with the theoretical distribution corresponding to a twinning fraction of 0.0 . Some other arbitrarily chosen data sets
(R. Huber, unpublished results) are displayed for comparison, including one for which twinning was suspected. As can be seen, the non-twinned experimental distribution curves do not coincide and lie above the theoretical values. However, for all of them the habiti are clearly exponential. Therefore, although this approach provides a clear indication of the presence of twinning in crystals, it does not give quantitative information.

\section{Initial structure determination}

Using the data set evaluated as space group $R 3$, an initial solution for the three molecules comprising the TC was obtained with the AMoRe package (Navaza, 1994). In fact, it was possible to independently determine the positions of PCPA, BPE and CTGC for the two twin species. The models used during the search were porcine procarboxypeptidase A (Guasch, Coll, Avilés \& Huber, 1992) for PCPA, porcine pancreatic elastase (Meyer, Cole, Radahakrishnan \& Epp, 1988) for BPE, and chymotrypsinogen A (Wang, Bode \& Huber, 1985) for CTGC.

The rotation function for PCPA (calculated for data between 15 and $4.0 \AA$ ) gave two clear prominent peaks at $\left(3.5,102.5,102.0^{\circ}\right)$ and $\left(116.2,77.3,282.2^{\circ}\right)$ (see Table 2 ). Both peaks are given in Eulerian angles. No further peaks appeared higher than $30 \%$ of the correlation coefficient value of the first one. For both rotations, a clear solution of the translation function was found in $\left(0.5127,0.3376,0.0\right.$; correlation coefficient $31.4 ; R_{\text {factor }}$ $42.4 \%)$ and $(0.3364,0.5083,0.0$; correlation coefficient $25.7 ; R_{\text {factor }} 43.8 \%$ ), respectively. The values are given in fractional coordinates. Both solutions are related by a $180^{\circ}$ rotation around the diagonal between $\mathbf{a}$ and $\mathbf{b}$ and obviously correspond to the solutions for each of the twin species. The first solution, giving the highest correlation, corresponds to the most abundant twin species. 
Table 2. Results of the Patterson search in twinned TC crystals

Dist. stands for the smallest distance (in $\AA$ ) between the centers of mass of the latter positioned molecule and the already fixed one(s) (denoted by $>$ ). The symmetry-equivalent positions are also considered. The results of the rotation function (RF; $\alpha, \beta, \gamma)$ are in Eulerian angles, those of the translation function (TF; $x, y, z$ ) in fractional cell coordinates. Corr. is the correlation coefficient (Navaza, 1994). The solution corresponding to the same searching model for both twin species do not appear simultaneously due to a restriction in the closest distance between two hypothetical solutions (20 ̊).

\begin{tabular}{|c|c|c|c|c|c|c|c|c|c|}
\hline $\begin{array}{r}\text { Molecule } \\
\text { Twin species } 1\end{array}$ & $\alpha$ & $\beta$ & $\gamma$ & $x$ & $y$ & $z$ & Corr. & $R_{\text {factor }}$ & Dist. \\
\hline \multirow{2}{*}{\multicolumn{10}{|c|}{$\begin{array}{l}\mathrm{RF} \text { and TF output for PCPA (11) } \\
\text { RF-peak-number (11) in output list: } 1 \text { st }\end{array}$}} \\
\hline & & & & & & & & & \\
\hline 11 & 3.5 & 102.5 & 102.0 & 0.5127 & 0.3376 & 0.0000 & 31.4 & 42.4 & \\
\hline \multicolumn{10}{|c|}{$\begin{array}{l}\mathrm{RF} \text { and } \mathrm{TF} \text { for BPE (12) with } 11 \text { fixed } \\
\text { RF-peak-number (12) in output list: 1st }\end{array}$} \\
\hline$>11$ & 3.5 & 102.5 & 102.0 & 0.5127 & 0.3376 & 0.0000 & 31.4 & 42.4 & \multirow[t]{2}{*}{51.0} \\
\hline 12 & 20.0 & 126.5 & 341.5 & 0.4532 & 0.0774 & 0.3137 & 41.0 & 39.5 & \\
\hline \multicolumn{10}{|c|}{$\begin{array}{l}\text { RF and TF for CTGC (13) with } 11 \text { and } 12 \text { fixed } \\
\text { RF-peak-number (13) in output list: 1st }\end{array}$} \\
\hline$>11$ & 3.5 & 102.5 & 102.0 & 0.5127 & 0.3376 & 0.0000 & 31.4 & 42.4 & \multirow{3}{*}{$\begin{array}{l}51.0 \\
42.8\end{array}$} \\
\hline$>12$ & 20.0 & 126.5 & 341.5 & 0.4532 & 0.0774 & 0.3137 & 41.0 & 39.5 & \\
\hline 13 & 28.3 & 129.7 & 179.9 & 0.7120 & 0.9173 & 0.0405 & 41.0 & 40.1 & \\
\hline \multicolumn{10}{|c|}{ Fast rigid-body refinement (fiting; Castellano, Oliva \& Navaza, 1992) of three positioned molecules } \\
\hline$>11$ & 3.0 & 103.1 & 101.9 & 0.5103 & 0.3371 & 0.0003 & 31.4 & 42.4 & 51.0 \\
\hline$>12$ & 19.2 & 125.1 & 339.4 & 0.4531 & 0.0770 & 0.3142 & 41.0 & 39.5 & 42.8 \\
\hline$>13$ & 29.4 & 128.0 & 180.9 & $0.7(196$ & 0.9202 & 0.0369 & 48.0 & 38.0 & 37.9 \\
\hline \multicolumn{10}{|c|}{ Twin species 2} \\
\hline \multirow{2}{*}{\multicolumn{10}{|c|}{$\begin{array}{l}\text { RF and TF output for PCPA (11) } \\
\text { RF-peak-number (11) in output list: } 2 \text { nd }\end{array}$}} \\
\hline & & & & & & & & & \\
\hline 11 & 116.2 & 77.3 & 282.2 & 0.3364 & 0.5083 & 0.0000 & 25.7 & 43.8 & \\
\hline \multirow{2}{*}{\multicolumn{10}{|c|}{$\begin{array}{l}\mathrm{RF} \text { and } \mathrm{TF} \text { for } \mathrm{BPE}(12) \text { with } 11 \text { fixed } \\
\text { RF-peak-number (12) in output list: } 11 \text { th }\end{array}$}} \\
\hline$>11$ & 116.2 & 77.3 & 282.2 & 0.3364 & 0.5083 & 0.0000 & & & \multirow{2}{*}{52.2} \\
\hline 12 & 98.4 & 53.2 & 159.9 & 0.7446 & 0.7855 & 0.0206 & 30.9 & 42.3 & \\
\hline \multicolumn{10}{|c|}{$\begin{array}{l}\text { RF and TF for CTGC (13) with } 11 \text { and } 12 \text { fixed } \\
\text { RF-peak-number (13) in output list: } 31 \text { st }\end{array}$} \\
\hline$>11$ & 116.2 & 77.3 & 282.2 & 0.3364 & 0.5083 & 0.0000 & 25.7 & 43.8 & \multirow{3}{*}{$\begin{array}{l}52.2 \\
43.0\end{array}$} \\
\hline$>12$ & 98.4 & 53.2 & 159.9 & 0.7446 & 0.7855 & 0.0206 & 30.9 & 42.3 & \\
\hline 13 & 92.3 & 50.6 & 0.9 & 0.5877 & 0.0419 & 0.2925 & 32.5 & 42.0 & \\
\hline \multicolumn{10}{|c|}{ Fast rigid-body refinement (fiting; Castellano, Oliva \& Navaza, 1992) of the three positioned molecules } \\
\hline 11 & 116.9 & 77.2 & 281.8 & 0.3371 & 0.5100 & -0.0003 & 25.7 & 43.8 & 52.2 \\
\hline 12 & 100.9 & 54.8 & 159.1 & 0.7441 & 0.7862 & 0.0198 & 30.9 & 42.3 & 43.0 \\
\hline 13 & 91.4 & 51.6 & 0.2 & 0.5864 & 0.0416 & 0.2944 & 34.0 & 41.5 & 37.9 \\
\hline
\end{tabular}

Fixing each one of the solutions obtained for PCPA, the searching procedure was repeated for the second (BPE) and the third (CTGC) molecule (see Table 2) composing the TC. For the whole trimeric complex, the correlations obtained for the most and less abundant twin species were $48.0\left(R_{\text {factor }} 38.0 \%\right)$ and $34.0\left(R_{\text {factor }}\right.$ $41.5 \%$ ) after fast rigid-body refinement ('fiting'; Castellano, Oliva \& Navaza, 1992; Navaza, 1994), respectively. It is interesting to remark that the smallest search model used (the chymotrypsinogen A molecule, for CTGC) represents only $28 \%$ of the asymmetric unit content and therefore less than $14 \%$ when the less abundant twin species is considered. An inspection of the packing on a graphic display showed no incompatible overlapping of the three positioned molecules within each of the twin domains.

\section{Determination of the twin fraction $\alpha$}

The twin fraction was determined from the highest correlation [see (6)] between observed and calculated intensities, the latter being obtained using the model for the more abundant twin species and (7).

$$
\begin{aligned}
\operatorname{Corr}(\alpha)= & \left(\sum _ { h k l } \left\{\left[I_{h k l}^{\text {calc-twin }}(\alpha)-\left\langle I_{h k l}^{\text {calc-twin }}(\alpha)\right\rangle\right]\right.\right. \\
& \left.\left.\times\left[I_{h k l}^{\text {obs }}-\left\langle I_{h k l}^{\text {obs }}\right\rangle\right]\right\}\right) /\left\{\sum _ { h k l } \left[I_{h k l}^{\text {calc-twin }}(\alpha)\right.\right. \\
& \left.\left.-\left\langle I_{h k l}^{\text {calc-twin }}(\alpha)\right\rangle\right]^{2} \sum_{h k l}\left(I_{h k l}^{\text {obs }}-\left\langle I_{h k l}^{\text {obs }}\right\rangle\right)^{2}\right\}^{1 / 2}
\end{aligned}
$$

where $\langle I\rangle$ is the mean intensity and,

$$
I_{h k l}^{\text {calc-twin }}(\alpha)=(1-\alpha) I_{h k l}^{\text {calc }}+\alpha I_{k h-l}^{\text {calc }} \text {. }
$$

The correlation coefficient has an unique maximum as a function of $\alpha$. The expression for the optimal twin fraction may be obtained by solving the equation $d \operatorname{Corr}(\alpha) / d \alpha=0$. We will derive it in a different way in order to discuss a more general method of detwinning, based on the work of Redinbo \& Yeates (1993). These authors had proposed the following model dependent estimate (8) of $I_{h k l}^{\mathrm{obs}-\text { theo }}$ (strictly speaking, they con- 
sidered the case $\alpha=0.5$ ) that has to be updated as the refinement proceeds,

$$
I_{h k l}^{\text {obs-theo }} \simeq\left[I_{h k l}^{\text {obs }}=\lambda \alpha I_{k h-l}^{\text {calc }}+\lambda(1-\alpha) I_{h k l}^{\text {calc }}\right] /[2 \lambda(1-\alpha)] .
$$

We first note the following relationship between the quadratic misfit and the correlation coefficient,

$$
\begin{aligned}
\operatorname{Min}_{\lambda} & \left\langle\left\{\left(I_{h k l}^{\text {obs }}-\left\langle I_{h k l}^{\text {obs }}\right\rangle\right)\right.\right. \\
& \left.\left.-\lambda\left[I_{h k l}^{\text {calc-twin }}(\alpha)-\left\langle I_{h k l}^{\text {calc-twin }}(\alpha)\right\rangle\right]\right\}^{2}\right\rangle \\
& =\left\langle\left(I_{h k l}^{\text {obs }}-\left\langle I_{h k l}^{\text {obs }}\right\rangle\right)^{2}\right\rangle\left[1-\operatorname{Corr}(\alpha)^{2}\right]
\end{aligned}
$$

Thus, instead of maximizing the correlation, we may minimize,

$$
\left\langle\left[C_{h k l}^{\text {obs }}-\lambda(1-\alpha) C_{h k l}^{\text {calc }}-\lambda \alpha C_{k h-l}^{\text {calc }}\right]^{2}\right\rangle,
$$

with respect to $\lambda$ and $\alpha$, where $C=I-\langle I\rangle$ denotes a 'centred' intensity. (10) may be considered as a quadratic expression in the new independent variables $\lambda(1-\alpha)$ and $\lambda \alpha$, hence it has an unique global minimum. The condition of minimum leads to a linear system whose solution gives,

$$
\begin{aligned}
\lambda= & \left\langle C_{h k l}^{\text {obs }} S_{h k l}^{\text {calc }}\right\rangle /\left\langle C_{h k l}^{\text {calc }} S_{h k l}^{\text {calc }}\right\rangle ; \\
\alpha= & {\left[\left\langle C_{h k l}^{\text {calc }} C_{h k l}^{\text {calc }}\right\rangle-\left(\left\langle C_{h k l}^{\text {calc }} S_{h k l}^{\text {calc }}\right\rangle\left\langle C_{h k l}^{\text {obs }} C_{h k l}^{\text {calc }}\right\rangle\right) /\left\langle C_{h k l}^{\text {obs }} S_{h k l}^{\text {calc }}\right\rangle\right] } \\
& \div\left[\left\langle C_{h k l}^{\text {calc }}\left(C_{h k l}^{\text {calc }}-C_{k h-l}^{\text {calc }}\right)\right\rangle\right],
\end{aligned}
$$

with $S_{h k l}^{\text {calc }}=C_{h k l}^{\text {obs }}+C_{k h-l}^{\text {calc }}$. If $\alpha$ is sensibly different from 0.5 , (1) and (2) can be solved for $I_{h k l}^{\text {obs-theo }}$,

$$
I_{h k l}^{\mathrm{obs}-\mathrm{theo}}=\left[(1-\alpha) I_{h k l}^{\mathrm{obs}}-\alpha I_{k h-l}^{\mathrm{obs}}\right] /(1-2 \alpha) .
$$

The correlation function displayed an unambiguous maximum at $\alpha=0.37$, a result that was insensitive to the resolution range chosen, and even to the number of positioned molecules used for the structure-factor calculation.

Once an accurate estimation of the twin fraction was available, the original data were deconvoluted using (12), rendering a final $R_{\text {symm }}$ of $12.9 \%$ and losing $19.4 \%$ of the measurements (due to missing $[h k l / k h-l]$ pairs) when applying the detwinning procedure to unmerged data, that is, before averaging the Friedel-mates. The initial $R_{\text {factor }}$ with this deconvoluted data is $41.8 \%$ for 11680 reflections in the resolution range between 9.0 and $3.5 \AA$, displaying the calculated map extra density that has allowed to, at least partially, improve the TC model.

The procedure here described was applied in order to systematically analyze the twinning fractions in TC crystals. A total of five data sets were processed in this way using the Patterson-search solutions found for the first crystal, rendering twinning fractions of $0.43,0.49$, and 0.43 , respectively. For the fifth, $\alpha$ is 0.04 , that is, the untwinned data obtained from this crystal will be used for the final refinement of the TC stucture.

\section{Concluding remarks}

We have described preliminary results concerning the crystal structure analysis of the ternary complex of procarboxypeptidase A, using hemihedrally twinned crystals. It was found that the estimation of the twin fraction was rather insensitive to the completeness of the model. Patterson search and detwinning may, thus, be fruitfully used to solve rather complicated structures.

The kind help provided by Dr K. H. Mann and Professor F. Lottspeich is gratefully acknowledged. This work was supported by grants PB92-0707, BIO92-0458, EMBO long-term fellowship ALTF 371-1992, and a fellowship from the Human Capital and Mobility Programme of the European Union (ERBCHBICT920053). The help provided by the Centre de Supercomputació de Catalunya (CESCA) is further acknowledged.

Note added in proof: the detwinning procedure in cases of hemihedral twinning described in this publication has been implemented as a sub-program called geminis in the AMoRe package.

\section{References}

Avilés, F. X., Vendrell, J., Guasch, A., Coll, M. \& Huber, R (1993). Eur. J. Biochem. 166, 379-385.

Bode, W. \& SCHirmer, T. (1985). Biol. Chem. Hoppe-Seyler, 366, 287-295.

Castellano, E., Oliva, G. \& Navaza, J. (1992). J. Appl. Cryst. 25 , 281-284.

Fisher, R. G. \& Sweet, R. (1980). Acta Cryst. A36, 755-760.

Guasch, A., Coll, M., Avilés, F. X. \& Huber, R. (1992). J. Mol. Biol. 244, 141-157.

Kerfelec, B., Cambillau, C., Puigserver, A. \& Chapus, C. (1986). Eur. J. Biochem. 157, 531-538.

Kiffersaufr, R., StetefFl.D, J., LotTSPeich, F. \& Hubfr, R. (1995). In preparation.

KоCH, E. (1992). International Tables for Crystallography, Vol. C, pp. 10-14. Dordrecht: Kluwer Academic Publishers.

LESLIE, A. G. W. (1991). Recent Changes to the MOSFLM Package for Processing Film and Image-Plate Data. CCP4 and ESF-EACMB Newsletter on Protein Crystallography. Warrington: SERC Daresbury Laboratory.

Matthews, B. W. (1968). J. Mol. Biol. 33, 491-497.

Meyer, E., Colf, G., Radahakrishinan, R. \& Epp, O. (1988). acta Cryst. B44, 26-38.

NavazA, J. (1994). Acta Cryst. A50, 157-163.

Pascual, R., Vendrell, J., Avilés, F. X., Bonicel, J., Wicker, C. \& PuigsfrVer, A. (1990). FEBS Lett. 277, 37-41.

Redinbo, M. R. \& Yeates, T. O. (1993). Acta Cryst. D49, 755-760.

ReES, D. C. (1980). Acta Cryst. A36, 578-581.

SteigemanN, W. (1991). PROTEIN. A Program System for the Crystal Structure Analysis of Proteins, Version 3.1, Max-Planck-Institut für Biochemie, Martinsried, München, Germany.

WANG, D., BodF, W. \& Huber, R. (1985). J. Mol. Biol. 185, 595-624. WILson, A. J. C. (1949). Acta Cryst. 2, 318-321. 